\title{
Beiträge zur Kenntniss der Microlepidopteren-Fauna der Erzherzogthümer 0esterreich ob und unter der Enns und Salzburgs.
}

Von Josef Mann in Wien.

(3. Fortsetzung.)

var. Proteana HS. 29. 30. -8 , in den Remisen auf dem Laaerberg.

Aspersana Hb. $259 .-7,8$, in jungen Laubhölzern, in den Remisen bei Tivoli, ziemlich selten.

Ferrugana Tr. -9 , in jungen Eichengebü schen in der Wiener Gegend, nicht selten. Raupe auf Quercus.

Lithargyrana HS. 23. - 7, 8, in jungen Eichengebüschen, selten. Raupe auf Quercus und Fagus.

Selasana HS. $370 .-7$, in den Remisen bei Tivoli, selten. Raupe auf Laubhölzern.

Quercinana $\mathbf{Z}$. $-5,6$, in den Remisen auf dem Laaerberg, bei Tivoli um Eichengebüsch. Raupe auf Quercus, minirtin den Biättern; (sehr häufig in Mehadia.)

Forskaleana L. -6 , überall in der Wiener Gegend auf Acer, nicht selten. Raupe auf Acer in zusammengesponnenen Blättern.

Holmiana L. $-6,7$, auf Crataegus Oxyacantha, nicht selten. Raupe auf Crataegus, Pyrus Malus et communis.

Contaminana Hb. 142. - 9, auf Crataegus- and wilden Rosa-Arten, nicht selten. Raupe auf denselben Stränchern.

var. Ciliana Hb. 171. - Gleichzeitig mit der Stammart und häufiger als diese.

\section{Tortrix Tr.}

Piceana L. $-6,7$, in Kiefernund Fichtenwaldungen, selten. Raupe auf Abies excelsa DC., Abies pecti. nata DC., Pinus sylvestris L. und Larix europaea DC.
Podana Sc. $-5,6$, auf jungen Quercus, Salix und Pyrus, nicht gar selten. Raupe auf Laubhölzern.

Crataegana Hb. 107. O (Roborana Hb. 126. 7 .) - 6, 7, in jungen Gebüschen in der Wiener Gegend, selten. Raupe auf Crataegus, Acer, Populus und Pyrus.

Xylosteana L. $-6,7$, im Prater und in der ganzen Wiener Gegend in jungen Gebüschen. Raupe auf Laubhölzern.

Rosana L. -6 , nirgends selten in Oesterreich, um Prunus spinosa und Crataegus. Raupe anf allerhand Sträuchern, auch auf Mentha aquatica und Aesculus Hippocastanum.

Sorbiana Hb. 113. $-6,7$, im Eichenwälḋchen bei Tivoli, am Laaerberg, Bisamberg etc. Raupe auf Quercus und Sorbus Aucuparia etc.

Semialbana Gn. $-6,7$, in Remisen bei Tivoli, in Mauer und Baden, um Hecken, selten.

Costana F. -7 , auf dem Bisamberge um Hecken, selten.

Corylana F. $-6,7$, überall in der Wiener Gegend in Gebüschen, nicht selten.

Ribeana Hb. 114. - 6, 7, überall in Gebüschen, nicht selten. Raupe auf Alnus und anderen Laubhölzern, auch auf Parietaria officinalis.

Cerasana Hb. $119 .-6,7$, im Prater und in anderen Wäldchen, nicht selten. Raupe auf Prunus spinosa, Populus und Pyrus.

Cinnamomeana Tr. -6 , auf dem Bisamberge, Liechtenstein und in Mauer, einzeln. Raupe auf Betula alba und Sorbus Aucuparia. 
Heparana Schiff. - 6, 7, überall um Wien in Gebüschen. Raupe anf Alnus, Salix, Acer etc., auch auf Parietaria officinalis.

Ab. Vulpisana HS. 34. - 7, einigemale im Prater auf Parietaria gefunden, selten. Raupe auf Laubhölzern.

Dumetana Tr. -7 , mehreremale am Liechtenstein aus Hecken gescheucht, selten.

Lecheana L. -6 , im Prater an Zaunplanken und auf Acer und Ulmus, nicht hänfig. Raupe auf Obstund anderen Bäumen.

Inopiana Hw. -7 , auf dem Alpeleck am Schneeberg, selten. Raupe auf niederen Pflanzen: Centaureen.

Histrionana Fröl. (Hb. 310. 11.) $6-8$, in der Wiener Gegend bei Mauer, in Fichtenwaldungen am Schneeberg. Raupe auf Abies excelsa DC.

Murinana Hb. 105. - 6, 7, in Tannenwäldern in der Wiener Gegend. Raupe auf Abies pectinata DC.

var. Immaculana Wachtl („Die Weisstannen-Triebwickler", pag. 15, Taf. III, Fig. 3) mit der Stammart, selten.

Musculana $\mathbf{H b} .-6,7$, auf Alnus, Acer etc., überall in der Wiener Gegend. Raupe auf verschiedenen Sträuchern und Laubhölzern.

Strigana $\mathbf{H b}$. - 5, 6, 8, überall in der Wiener Gegend auf Hutweiden und Bergwiesen. Raupe auf Artemisia campestris.

Diversana Hb. - 6, im Prater und an anderen Orten in Gebüschen und Remisen, auch auf Pyrus Malus. Raupe auf Sträuchern und Bäumen.

Ochreana Hb. 134. - 5, 6, von Mauer bis Vöslau, Emmerberg auf Berglehnen.

Politana Hw. -5 und 8, in der Wiener Gegend zwischen jungen Pinus.

Cinctana Schiff. - 5, 6, 8, überall in der Wiener Gegend auf trockenen Wiesen und Bergen. Raupe an niederen Pflanzen, Thymus n. s. w.
Asinana Hb. -6 , im Mauererwalde von Quercus abgeklopft, sehr selten, auch im Prater.

Rigana Sodof. - $4-9$, überall auf Bergen in der Umgebung von Wien, nicht selten. Raupe in röhrenförmigen Gängen an Anemonen.

0xyacanthana HS. -6 , im Prater auf Weissdornbäumen nnd an Zaunplanken, selten. Raupe April in jungen Trieben von Crataegus Oxyacantha.

Ministrana L. - 5, 6, in Erlengebüschen im Prater, nicht selten. Raupe auf Almus, Salix, Betula etc.

Bifasciana $\mathbf{H b} .-5,6$, Mauer auf jungen Quercus, selten.

Conwayana F. -6 , in den Remisen bei Tivoli, Laaerwald, Mauer, Bisamberg in Gebüschen.

Bergmanniana L. -6 , um wilde Rosenhecken überall um Wien, auch auf Alpen: Hohe Wand. Raupe auf Rosa in den Knospen und jungen Trieben.

Loeflingiana L. $-5,6$, überall um Wien in Eichengebüschen, nicht selten. Raupe auf Quercus.

Viridana L. Ratz. - 6, überall um Wien in Eichengebüschen, hänfig. Raupe auf Quercus-Arten, oft sehr schädlich.

Forsterana F. (Adjunctana Tr. F. R. 9. 1.) $-6,7$, Mauer und auf dem Schneeberge um Lärchenbäume. Raupe anf Vaccinium Myrtillus und V. Vitis Idaea.

Viburniana F. -6 , überall in der Mödlinger und Badener Gegend anf Berglehnen in jungen Hecken. Raupe an Ledum palustre, Andromeda, Chrysanthemum.

Unicolorana Dup. - 7, auf der Heaplacke des Schneeberges, sehr selten.

Paleana Hb. -6 , in den Praterauen spät Abends zu finden, nicht mit Flavana Hüb. zu verwechseln. Raupe auf Alnus und Salix.

var. Icterana Froel. - 7, auf dem Schneeberge auf der Heuplacke, Kuh- 
schneeberg bei Tags immer zu finden. Raupe an Quercus und Vaccinium.

Steineriana Hb. - 7, auf der Heuplacke am Schneeberge, selten. Raupe auf den Blüthen verschiedener Alpenpflanzen, nach Herrn v. Hornig insbesondere auf Buphthalmum salicifolium.

Rusticana Tr. $-4,5$, überall in der Wiener Gegend anf Wiesen und grasreichen Berglehnen, häufig. Raupe auf verschiedenen niederen Pflanzen.

Rolandriana L. - 6, Gahns, auf der Bodenwiese, nicht selten.

Reticulana Hb. 271. - 6, im Prater auf Erlen, nicht gar selten. Raupe auf Alnus.

Pilleriana Schiff. -7 , in der Wiener Gegend von Mauer bis Baden. Raupe auf Vitis vinifera und noch anf vielen anderen niederen Pflanzenarten gezogen.

Grotiana F. - 6, 7, bei Tivoli, am Laaerberg in den Remisen, in der Badener Gegend, selten. Raupe auf Quercus und Crataegus.

Gnomana Cl. - 7, um Wien in jungen Gebüschen.

Gerningana Schiff. $-6,8$, auf Berglehnen und Wiesen, auch auf den Schneeberger Alpen, nicht selten.

Rhombicana HS. -6 , Neusteinhof und Himmelswiese bei Kalksburg, in grasreichen Gräben und an Rändern, selten. Raupe auf jungen Astern und anderen niederen Pflanzen in den Herzblättern.

Prodromana Hb. - 4, bei Salmansdorf, Bisamberg, 5, Eichkogl, sehr selten. Raupe auf Potentilla anserina. Herr v. Hornig erzog dieselbe von wild wachsendem Daucus Carota.

Favillaceana $\mathbf{H b}$. -5 , in Mauer, 6 auf dem Gahns um junge Abies ex. celsa und Pinus. Raupe auf Erica, auch auf Rubus Idaeus.

\section{Sciaphila Tr.}

Osseana Sc. - 7, 8, auf den Schneeberger Alpen, nicht selten.
Argentana Cl. - 7, Höllenthal, Gahns, Saugraben am Schneeberg, nicht selten.

Penziana Hb. 85. - 7, Höllenthal, an Felsen, Alpeleck, Saugraben, hohe Wand.

var. Styriacana HS. 119 . -7 , bei Mödling, Gumpoldskirchen etc. an Mauern, nicht selten.

Chrysantheana Dup. - 6, 7, überall in der Wiener Gegend, nicht häufig an Zäunen und Planken. Raupe auf niederen Pflanzen; Chenopodium, Scabiosa etc. (Raupe mit schwarzem Kopf, Warzen weiss.)

Wahlbomiana L. $-6,7$, nirgends selten in Oesterreich. Raupe auch auf niederen Pflanzen. (Raupe mit honiggelbem Kopf, Warzen schwarz.)

var. Alticolana HS. 112. $-\%$, auf den Schneeberger Alpen meist auf Larix europaea und Abies excelsa DC. Raupe auf niederen Pflanzen. (Raupe: Kopf hellgelb.)

var. Virgaureana $\mathrm{Tr}$. -7 , in Gebüschen und an Planken, selten. Raupe auf Solidago Virgaurea. (Raupe plump, Kopf und Warzen gelb.)

var. Minorana HS. 104-6. (Var. Incertana Tr., H. S. 121. 2.) - 6, 7, überall in Gebüschen auf Bäumen und Zaunplanken, nicht selten. Raupe auf Crataegus, Acer und niederen Pflauzen.

var. Communana HS. $-6,7$, in den Praterauen, sowie in Berggebüschen. Raupe auf Laubhölzern und niederen Pflanzen.

Pasivana Hb 99. - 6, im Prater an Zäunen und Gebüschen, in den Remisen bei Tivoli, in Mauer etc. Raupe auf niederen Pflanzen.

Abrasana Dup. HS. 99. - 6, überall auf Ahornbäumen in der Wiener Gegend, nicht selten. Raupe auf Acer in zusammengesponnenen Blättern gefunden, jedoch auf Achillea Millefolium häufiger. 
Nubilana Hb. 111. - 6, nicht selten auf Crataegus, Prumus spinosa et domestica, wo die Raupe lebt.

\section{Sphaleroptera Gn.}

Alpicolana Hb. 328. - 8, auf der Raxalpe, Schneekoppe, Schneeberggipfel, sehr selten.

\section{Doloploca $\mathrm{Hb}$.}

Punctulana Schiff. - 4, 5, Wiener Gegend in Remisen und Gebüschen, selten.

\section{Chimatophila Stph.}

Tortricella Hb. Tin. 11 - 4, 5, äberall um Wien in Eichen- und Buchengebüschen, nicht selten. Raupe auf Quercus und Fagus.

\section{Exapate $\mathrm{Hb}$.}

Congelatella $\mathrm{Cl}-10,11$, auf Schleben und Pflaumenbäumen, in Obstgärten. Raupe meist auf Prunus domestica, Ligustrum, Crataegus, Corylus und Rubus fruticosus.

\section{Olindia Gn.}

Hybridana $\mathbf{H b}$. 238. $-6,7$, in der Wiener Gegend um Dornhecken und Gesträuch, nicht gar selten. Raupe auf Laubgebüschen.

var. Albulana Tr. - 7, im Guttensteinerthal und der Voit an Berglehnen auf Vaccinium and Erica.

UImana Hb. 278. $\sigma^{\prime}$ (Areolana $\mathrm{Hb}$. 279. ) - 7, Gahns, auf der Brandstattwiese, am Alpeleck, auf Rubus Idaeus.

\section{Cochylis Tr.}

Parreyssiana Dup. - 6, auf dem Eichkogl, Baden ober dem Kalvarienberge, sehr selten.

Hamana L. - 6, in der Wiener Gegend anf grasreichen Plätzen, besonders um Felder mit Pisum und Vicia, nicht selten. Raupe in Hülsen der Leguminosen, oft sehr schädlich, ferner auf Trifolium-Arten.
Zoegana L. Hb. 138. (Hamana Cl.) 6, auf Hutweiden, grasreichen Berglehnen und Bergen. Raupe in Wurzeln der Scabiosen.

Amiantana Hb. 155. $-6,7$, von Mauer bis Baden auf grasreichen Berglehnen und Bergen, selten. Raupe auf niederen Pflanzen.

Zebrana Hb. 197. - 6, einmal auf dem Bisamberge gefangen, sehr selten.

Perfusana Gn. - 6, 7, auf den Schneeberger Alpen, selten. Raupe auf niederen Pflanzen, Centaurea.

Purgatana $\mathrm{Tr}$. $-4,5$, überall in der Wiener Gegend auf Berglehnen und Bergen, nicht selten. Raupe auf Artemisia campestris.

Schreibersiana Fröl. - 5, 6, im Prater auf Ulmus, richt selten. Raupe auf Ulmus und Acer; Verpuppung unter der Rinde.

Cruentana Fröl. - 7, Schneeberger Alpen, Gahns, Alpelek, selten.

Ambiguelia Hb. (Roserana Fröl.) 5,8 , in Weingärten überall in der Wiener Gegend. Raupe sehr schädlich den Blüthen und Früchten von Vitis vinifera.

Straminea $\mathrm{Hw} .-5,6$, überall um Wien anf Berglehnen und Bergen. Raupe anf Centaurea und Artemisia.

Hilarana HS. - 7, auf dem Alpensteig zum Kaiserbrunnen hinab, dann im Saugraben, sehr selten.

Dipoltella $\mathrm{Hb} .-7$, im Prater, in Mauer, Rodaun etc. an Bächen, auf Berglehnen und Bergen. Raupe auf Camillenblüthen.

Zephyrana Tr. et Variet. $-4-8$, überall in der Wiener Gegend auf Hutweiden und Berglehnen, nicht selten.

Rutilana Hb. 249. - 6, 7, anf dem Bisamberg und den Schneeberger Alpen um Juniperus. Raupe auf Juniperus.

Aurofasciana Mn. - 7, Gahns, anf der Bodenwiese, 1879. (Fortsetzung folgt.) 


\section{$2 \mathrm{BHL}$ Biodiversity Heritage Library}

Mann, Josef Johann. 1884. "Beiträge zur Kenntniss der

Microlepidopteren-Fauna der Erzherzogthümer Oesterreich ob und unter der Enns und Salzburgs. (Anm.: 3. Fortsetzung)." Wiener entomologische Zeitung 3, 273-276. https://doi.org/10.5962/bhl.part.13856.

View This Item Online: https://www.biodiversitylibrary.org/item/43792

DOI: https://doi.org/10.5962/bhl.part.13856

Permalink: https://www.biodiversitylibrary.org/partpdf/13856

\section{Holding Institution}

Smithsonian Libraries

\section{Sponsored by}

Smithsonian

\section{Copyright \& Reuse}

Copyright Status: NOT_IN_COPYRIGHT

This document was created from content at the Biodiversity Heritage Library, the world's largest open access digital library for biodiversity literature and archives. Visit BHL at https://www.biodiversitylibrary.org. 